\title{
A New Bound on the Local Density of Sphere Packings
}

\author{
Douglas J. Muder
}

The MITRE Corporation, Bedford, MA 01730, USA

doug@mitre.org

\begin{abstract}
It is shown that a packing of unit spheres in three-dimensional Euclidean space can have density at most $0.773055 . .$. , and that a Voronoi polyhedron defined by such a packing must have volume at least $5.41848 \ldots$. These bounds are superior to the best bounds previously published [5] ( 0.77836 and 5.382, respectively), but are inferior to the tight bounds of $0.7404 \ldots$ and $5.550 \ldots$ claimed by Hsiang [2].

Our bounds are proved by cutting a Voronoi polyhedron into cones, one for each of its faces. A lower bound is established on the volume of each cone as a function of its solid angle. Convexity arguments then show that the sum of all the cone volume bounds is minimized when there are 13 faces each of solid angle $4 \pi / 13$.
\end{abstract}

\section{Introduction}

The density of a sphere packing in a space is the percentage of the volume of the space that lies inside the spheres. For spaces of infinite volume the density is defined as a limit of the densities associated with a set of nested, convex, finite subsets whose union is the entire space. In three-dimensional Euclidean space the vertices of the face-centered cubic lattice can be used to define the cannonball packing, a packing of nonoverlapping unit spheres whose density is $\pi / \sqrt{18} \sim$ $0.7404 \ldots$... This packing was known to Kepler, who conjectured that it was the best possible.

Over the years a number of upper bounds on the density of nonoverlapping packings of unit spheres in three dimensions have been established, most recently 0.7796 by Rogers [6], 0.77844 by Lindsey [3], and 0.77836 by Muder [5]. Since [5], Lindsey [4] has announced a bound of 0.7736 and Hsiang [2] has claimed to have proved Kepler's conjecture. As of this writing, the status of these claims is unresolved. A good general reference on sphere packings and what is known about them is [1].

Each sphere in a packing has a Voronoi polyhedron associated with it. This is 
the set of points in the space that lie closer to the center of that sphere than to the center of any of the other spheres. The Voronoi polyhedra decompose the space, and if the spheres are nonoverlapping each polyhedron contains its associated sphere. By convention, the "center" of a Voronoi polyhedron is the center of its associated sphere. The ratio of the volume of a sphere to the volume of its Voronoi polyhedron is a local measure of density, and it is well known that any upper bound on the local densities in a sphere packing is also an upper bound on the density of the packing as a whole. In this paper we prove

Theorem. A Voronoi polyhedron defined by a packing of unit spheres in three dimensions has volume at least $13 \beta(4 \pi / 13) \sim 5.41848 \ldots$, where $\beta(\psi)$ is defined at $\psi=4 \pi / 13$ by the parametric equations

$$
\begin{aligned}
& \beta_{r}=\frac{5 r \sqrt{1-2 r^{2}}}{3 \sqrt{2}}+\frac{1}{6}\left(\pi-5 \arctan \sqrt{\frac{1-2 r^{2}}{2 r^{2}}}\right) \\
& \psi_{r}=2\left(\pi-5 \arctan \sqrt{\frac{1-2 r^{2}}{3 r^{2}}}\right)-\sqrt{\frac{8}{3}}\left(\pi-5 \arctan \sqrt{\frac{1-2 r^{2}}{2 r^{2}}}\right) .
\end{aligned}
$$

From this theorem immediately follows

Corollary. The density of a packing of unit spheres in three dimensions is at most

$$
\frac{4 \pi}{39 \beta(4 \pi / 13)} \sim 0.773055 \ldots
$$

The proof of our theorem is easy to describe: For each face $\mathscr{F}$ of a Voronoi polyhedron with center $A$, construct the cone with base $\mathscr{F}$ and apex $A$. The sum of the volumes of these facecones is the volume of the polyhedron, and the sum of their solid angles at $A$ is $4 \pi$. We find a function $\beta(\psi)$ such that any of the facecones whose solid angle is $\psi$ has volume at least $\beta(\psi)$. We then use convexity arguments to show that the sum $\sum \beta\left(\psi_{i}\right)$ is minimized subject to the constraint $\sum \psi_{i}=4 \pi$ when there are 13 identical $\psi_{i}$ all equal to $4 \pi / 13$. This minimum is then our bound.

\section{Structure of the Proof}

Our proof has three major steps, summarized by Propositions A, B, and C. In this section we state these propositions and show how the theorem is proved from them. Sections 3, 4, and 5 prove these propositions based on Technical Lemmas TL1-TL8 proved in Section 6.

We get our bounds on the volumes of facecones by comparing them with right circular cones and shaved right circular cones of the same solid angle. First let us 
describe these objects. A right circular cone (RCC) is a cone whose base is a disk and whose apex lies on a ray which is perpendicular to the plane of the disk and originates at the center of the disk. The distance from the apex to the center of the disk is the height of the RCC and its radius is the radius of the disk. Consider a disk of center $M$ and a set of chords of its boundary circle. The shaved circle defined by the chords is the set of all points $P$ of the disk such that the segment $\overline{M P}$ does not intersect the chords. (Equivalently, it can be thought of as the intersection of the disk with a polygon.) The number of chords is the order of the shaved circle. We say that the shaved circle is vertex-free if the defining chords do not intersect. It is regular of order $n$ if it is defined by $n$ nonintersecting chords of the same length. A shaved right circular cone (SRCC) is a cone whose base is a shaved circle and whose apex lies on a ray which is perpendicular to the plane of the disk and originates at the center of the disk. Its height is defined in the same way as that of an RCC. An SRCC has the same order as its base and is regular or vertex-free if its base is. The distance from center of the disk to the nearest chord is called the inner radius of the SRCC, and the radius of the disk is called the outer radius. An RCC can be thought of as a regular SRCC of any order with inner and outer radii equal.

The definition of an SRCC is motivated by the following considerations: In [5] it was shown that the most efficient faces for constructing Voronoi polyhedra of small volume are regular pentagons. (An ideally efficient face would be circular, have a small radius, and be as close to the center of the polyhedron as possible. The pentagonal faces of [5] are the nearest possible approximation of this.) Faces of six or more sides are difficult to handle analytically, but are less efficient than pentagons because at most five edges of a face of a Voronoi polyhedron can be close to its center. In this paper we analyze facecones by replacing them with SRCCs of the same solid angle but less volume. Our construction process allows us to make do with SRCCs of order at most five, since at most five edges of the original face fall within the outer radius of the SRCC. In this way many of the difficulties of [5] are avoided.

Facecones are replaced by SRCCs of four basic types, depending on the solid angle of the facecone. We partition the interval $[0,2 \pi)$ of possible solid angles by

$$
0<\hat{\psi}_{1}<\hat{\psi}_{2}<\hat{\psi}_{3}<2 \pi
$$

where

$$
\begin{aligned}
& \hat{\psi}_{1}=\frac{2}{3}(3-\sqrt{8}) \pi \sim 0.3593 \\
& \hat{\psi}_{2}=10 \sqrt{\frac{2}{3}} \arctan \left(\frac{1}{\sqrt{2}}\right)-\frac{2 \sqrt{6}-1}{3} \pi \sim 0.9424 \\
& \hat{\psi}_{3}=\frac{2}{\sqrt{3}}(\sqrt{3}-\sqrt{2}) \pi \sim 1.1530
\end{aligned}
$$


This partitioning seems unintuitive, but is based on simple geometric considerations. There are three easily bounded constants associated with each face: its height, the distance from the polyhedron's center to the face's nearest edge, and the distance from the polyhedron's center to the face's nearest vertex. These give us the height, inner radius, and outer radius of our SRCCs. Every facecone must have height $h \geq 1$, and facecones of small solid angle are possible only for larger heights, with arbitrarily small solid angles possible only for $h \geq \sqrt{2}$. In our SRCC construction process, $\hat{\psi}_{2}$ is the smallest solid angle that occurs with $h=1$. (This is what causes the convexity of $\beta(\psi)$ to change at $\hat{\psi}_{2}$, making this the most efficient solid angle for an SRCC. The solid angle $4 \pi / 13$ appears in the theorem because this is the smallest unit fraction of $4 \pi$ that is greater than $\hat{\psi}_{2}$.) Our bounds on the distances of edges and vertices become equal at $h=2 / \sqrt{3}$, corresponding to an RCC of solid angle $\hat{\psi}_{1}$. For solid angles greater than $\hat{\psi}_{2}$, our bounding SRCCs have height 1 , outer radius $1 / \sqrt{2}$, and an inner radius that increases with solid angle. Inner and outer radii become equal at $\hat{\psi}_{3}$, the solid angle of an RCC of height 1 and radius $1 / \sqrt{2}$.

This replacing of facecones with SRCCs is given by

Proposition A. A facecone of solid angle $\psi$ has volume at least that of an SRCC of solid angle $\psi$ of the form:

(1) An $R C C$ of height $h \in[2 / \sqrt{3}, \sqrt{2}]$ and radius $\left(2-h^{2}\right) / \sqrt{4-h^{2}}$ for $\psi \in\left[0, \hat{\psi}_{1}\right]$.

(2) A regular, vertex-free, order-5 SRCC of height $h \in[1,2 / \sqrt{3}]$, outer radius $\sqrt{\frac{3}{2}-h^{2}}$, and inner radius $\left(2-h^{2}\right) / \sqrt{4-h^{2}}$ for $\psi \in\left[\hat{\psi}_{1}, \hat{\psi}_{2}\right]$.

(3) A regular, vertex-free, order-5 SRCC of height 1 , outer radius $1 / \sqrt{2}$, and inner radius $r \in[1 / \sqrt{3}, 1 / \sqrt{2}]$ for $\psi \in\left[\hat{\psi}_{2}, \hat{\psi}_{3}\right]$.

(4) An $R C C$ of height 1 and radius $R \geq 1 / \sqrt{2}$ for $\psi \in\left[\hat{\psi}_{3}, 2 \pi\right)$.

Using Technical Lemma TL1 to evaluate the volumes and solid angles of the objects listed in Proposition A allows us to state a more analytic version of the proposition. This version defines $\beta(\psi)$ parametrically on the four subintervals of $[0,2 \pi)$. A graph of $\beta(\psi)$ is given in Fig. 1.

Proposition $\mathbf{A}^{\prime}$. A facecone of solid angle $\psi$ has volume at least $\beta(\psi)$ where $\beta(\psi)$ is defined by the following parametric equations:

$$
\begin{aligned}
& \beta_{h}=\frac{\pi h\left(2-h^{2}\right)^{2}}{3\left(4-h^{2}\right)} \\
& \psi_{h}=\pi\left(2-h \sqrt{4-h^{2}}\right)
\end{aligned}
$$

for $h \in[2 / \sqrt{3}, \sqrt{2}]$, in which case $\psi_{h}$ parametrizes all values in $\left[0, \hat{\psi}_{1}\right]$ 


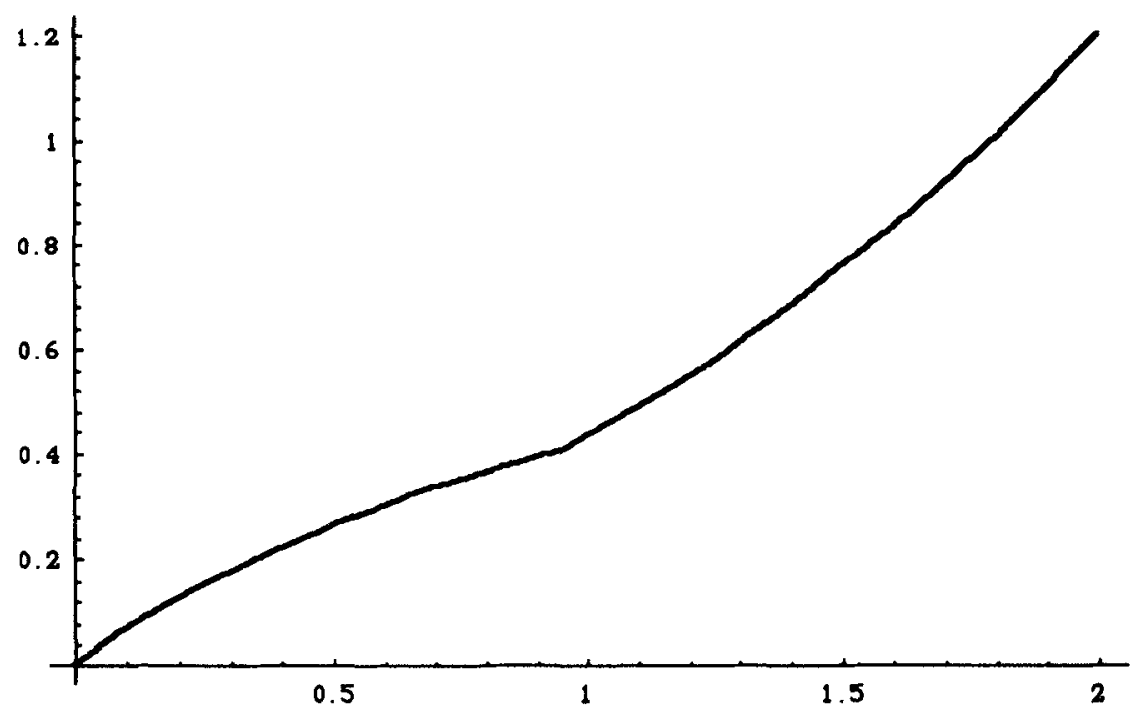

Fig. 1. Graph of $\beta(\psi)$.

$$
\begin{aligned}
& \beta_{h}=\frac{5 h\left(2-h^{2}\right) \sqrt{4-3 h^{2}}}{3 \sqrt{2}\left(4-h^{2}\right)}+\frac{h\left(3-2 h^{2}\right)}{6}\left(\pi-5 \arctan \frac{\sqrt{4-3 h^{2}}}{\sqrt{2}\left(2-h^{2}\right)}\right), \\
& \psi_{h}=2\left(\pi-5 \arctan \frac{h \sqrt{4-3 h^{2}}}{\sqrt{3\left(2-h^{2}\right)}}\right)-2 \sqrt{\frac{2}{3}} h\left(\pi-5 \arctan \frac{\sqrt{4-3 h^{2}}}{\sqrt{2}\left(2-h^{2}\right)}\right)
\end{aligned}
$$

for $h \in[1,2 / \sqrt{3}]$, in which case $\psi_{h}$ parametrizes all values in $\left[\hat{\psi}_{1}, \hat{\psi}_{2}\right]$;

$$
\begin{aligned}
& \beta_{r}=\frac{5 r \sqrt{1-2 r^{2}}}{3 \sqrt{2}}+\frac{1}{6}\left(\pi-5 \arctan \sqrt{\frac{1-2 r^{2}}{2 r^{2}}}\right), \\
& \psi_{r}=2\left(\pi-5 \arctan \sqrt{\frac{1-2 r^{2}}{3 r^{2}}}\right)-2 \sqrt{\frac{2}{3}}\left(\pi-5 \arctan \sqrt{\frac{1-2 r^{2}}{2 r^{2}}}\right)
\end{aligned}
$$

for $r \in[1 / \sqrt{3}, 1 / \sqrt{2}]$, in which case $\psi_{r}$ parametrizes all values in $\left[\hat{\psi}_{2}, \hat{\psi}_{3}\right]$;

$$
\begin{aligned}
& \beta_{R}=\frac{\pi R^{2}}{3}, \\
& \psi_{R}=2 \pi\left(1-\frac{1}{\sqrt{R^{2}+1}}\right)
\end{aligned}
$$

for $R \geq 1 / \sqrt{2}$, in which case $\psi_{R}$ parametrizes all values in $\left[\hat{\psi}_{3}, 2 \pi\right)$. 


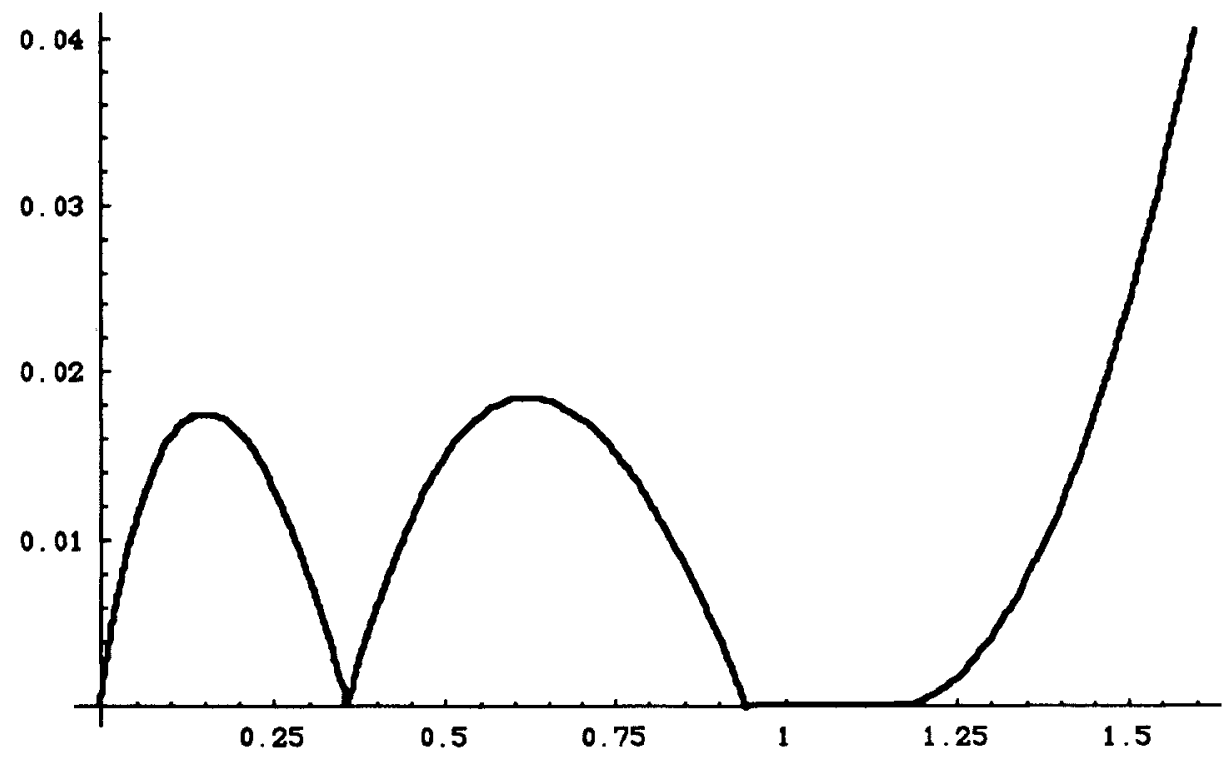

Fig. 2. Graph of $\beta(\psi)-L(\psi)$.

As might be imagined from its description above, $\beta(\psi)$ is rather difficult to work with. In Proposition B we bound $\beta(\psi)$ below with a function $L(\psi)$ which is linear except in the critical region between $\hat{\psi}_{2}$ and $\hat{\psi}_{3}$, where exactness is important. Numerical evidence for Proposition B is given in Fig. 2.

Proposition B. Let

$$
L(\psi)= \begin{cases}\frac{\beta\left(\hat{\psi}_{1}\right)}{\hat{\psi}_{1}} \psi & \text { for } \psi \in\left[0, \hat{\psi}_{1}\right], \\ \beta\left(\hat{\psi}_{1}\right)+\frac{\beta\left(\hat{\psi}_{2}\right)-\beta\left(\hat{\psi}_{1}\right)}{\hat{\psi}_{2}-\hat{\psi}_{1}}\left(\psi-\hat{\psi}_{1}\right) & \text { for } \psi \in\left[\hat{\psi}_{1}, \hat{\psi}_{2}\right], \\ \beta(\psi) & \text { for } \psi \in\left[\hat{\psi}_{2}, \hat{\psi}_{3}\right], \\ \beta\left(\hat{\psi}_{3}\right)+\beta^{\prime}\left(\hat{\psi}_{3}\right)\left(\psi-\hat{\psi}_{3}\right) & \text { for } \psi \in\left[\hat{\psi}_{3}, 2 \pi\right) .\end{cases}
$$

Then $\beta(\psi) \geq L(\psi)$ for all $\psi \in[0,2 \pi)$.

The problem is now a purely analytic one which is handled by

Proposition C. Suppose $\psi_{i} \in[0,2 \pi)$ for every $i$, and $\sum_{i=0}^{N} \psi_{i}=4 \pi$. Then

$$
\sum_{i=0}^{N} L\left(\psi_{i}\right) \geq 13 L\left(\frac{4 \pi}{13}\right)
$$


Proof of the Theorem. Let $\mathscr{V}$ be a Voronoi polyhedron defined by a packing of unit spheres. Suppose it has $N$ faces, and that the facecones have solid angles $\psi_{1}, \ldots, \psi_{N}$. By Proposition $\mathrm{A}^{\prime}$ we know that the volume of $\mathscr{V}$ is at least $\sum_{i=1}^{N} \beta\left(\psi_{i}\right)$. Now Propositions B, C, and the definition of $L$ allow us to conclude

$$
\sum_{i=1}^{N} \beta\left(\psi_{i}\right) \geq \sum_{i=1}^{N} L\left(\psi_{i}\right) \geq 13 L\left(\frac{4 \pi}{13}\right)=13 \beta\left(\frac{4 \pi}{13}\right)
$$

\section{Proof of Proposition A}

The proof of Proposition A has three main steps, corresponding to Propositions $\mathrm{A} 1, \mathrm{~A} 2$, and A3. In this section we prove these propositions in terms of the technical lemmas (which are found in Section 6) and show how they are used to prove Proposition A.

If $\mathscr{R}$ is a planar region and $A$ is a point out of the plane, then let $\mathscr{C}_{A}(\mathscr{R})$ be the cone with base $\mathscr{R}$ and apex $A$. In situations where $\mathscr{R}$ is a region in the plane of a face of a Voronoi polyhedron and $A$ is the center of the polyhedron, we simplify the notation and write $\mathscr{C}(\mathscr{R})$.

If $\mathscr{S}$ and $\mathscr{S}^{\prime}$ are shaved circles in the same plane, with a common center $M$, and $A$ is a point on the line perpendicular to the plane at $M$, we say that the SRCC $\mathscr{C}_{A}(\mathscr{P})$ is replaceable by $\mathscr{C}_{A}\left(\mathscr{S}^{\prime}\right)$ if $\mathscr{C}_{A}(\mathscr{S})$ and $\mathscr{C}_{A}\left(\mathscr{P}^{\prime}\right)$ have the same apex solid angle and $\operatorname{vol}\left(\mathscr{C}_{A}(\mathscr{P})\right) \geq \operatorname{vol}\left(\mathscr{C}_{A}\left(\mathscr{S}^{\prime}\right)\right)$. The replacement process is guided by a simple intuition (expressed rigorously in TL3): In order for a cone to have a small volume in comparison with its apex solid angle, the height $h$ should be small and the area of the base should be concentrated around the foot of the perpendicular from the apex to the plane of the base. This motivates the process of constructing an SRCC to replace a given facecone $\mathscr{C}(\mathscr{F})$. We begin by drawing a circle of radius $\sqrt{\frac{3}{2}-h^{2}}$ around the foot $M$ of the perpendicular from the apex. This radius is small enough to ensure that all vertices and all but five edges of $\mathscr{F}$ lie outside the circle. The intersection of the corresponding disk with $\mathscr{F}$ is a shaved circle and defines an SRCC. We account for the solid angle outside the circle by increasing the inner radius of the SRCC, effectively moving area from outside the circle to inside the circle, thereby improving the concentration of area around $M$. If the inner radius reaches the outer radius without accounting for all of the solid angle, we expand both, creating an RCC of radius greater than $\sqrt{\frac{3}{2}-h^{2}}$. Of all the SRCCs that can be constructed in this way from facecones of a given solid angle, we then choose the one of minimum height, which also turns out to be the one of minimum volume.

Proposition A1. Any facecone $\mathscr{C}(\mathscr{F})$ of height $h \geq 2 / \sqrt{3}$ is replaceable by either

(1) an $R C C$ of height $\bar{h} \in[2 / \sqrt{3}, \min (h, \sqrt{2})]$ and radius $\left(2-\bar{h}^{2}\right) / \sqrt{4-\bar{h}^{2}}$; or

(2) an $R C C$ of height $2 / \sqrt{3}$ and radius $R>1 / \sqrt{6}$. 
Proof. Let $\mathscr{B}$ be a circle in the plane of $\mathscr{F}$, with center $M$ and radius $R$ such that $\mathscr{C}(\mathscr{B})$ and $\mathscr{C}(\mathscr{F})$ have the same solid angle.

It follows that $\mathscr{C}(\mathscr{F}-\mathscr{B})$ and $\mathscr{C}(\mathscr{B}-\mathscr{F})$ have the same solid angle, which we denote $\varphi$. However, all points of $\mathscr{F}-\mathscr{B}$ are at least $\rho=\sqrt{R^{2}+h^{2}}$ from $A$, while all points of $\mathscr{B}-\mathscr{F}$ are at most $\rho$ from $A$. Therefore TL3 allows us to deduce

$$
\begin{aligned}
\operatorname{vol}(\mathscr{C}(\mathscr{F}))-\operatorname{vol}(\mathscr{C}(\mathscr{B})) & =\operatorname{vol}(\mathscr{C}(\mathscr{F}-\mathscr{B}))-\operatorname{vol}(\mathscr{C}(\mathscr{B}-\mathscr{F})) \\
& \geq \varphi \frac{\rho^{3}}{3}-\varphi \frac{\rho^{3}}{3} \\
& =0 .
\end{aligned}
$$

So we see that $\mathscr{C}(\mathscr{F})$ is replaceable by $\mathscr{C}(\mathscr{B})$.

For each $\bar{h} \in[2 / \sqrt{3}, h]$ let $R C C(\bar{h})$ be the $\mathrm{RCC}$ of height $\bar{h}$ and radius $R_{\bar{h}}=R \bar{h} / h$. The cone $R C C(\bar{h})$ is similar to $\mathscr{C}(\mathscr{B})$ but smaller and so has the same solid angle as $\mathscr{C}(\mathscr{B})$ but less volume. So $\mathscr{C}(\mathscr{B})$ is replaceable by any $R C C(\bar{h})$.

By TL4, either $h>\sqrt{2}$ or $\mathscr{F}$ contains a circle of radius $\left(2-h^{2}\right) / \sqrt{4-h^{2}}$ about $M$. Therefore we know $R>g(h)$, where

$$
g(h)= \begin{cases}\frac{\left(2-h^{2}\right)}{\sqrt{4-h^{2}}} & \text { for } h \leq \sqrt{2} \\ 0 & \text { for } h>\sqrt{2} .\end{cases}
$$

As $\bar{h}$ decreases so does $R_{\bar{h}}$, but $g(\bar{h})$ increases. Therefore either there is some $\bar{h} \in[2 / \sqrt{3}, \min (\sqrt{2}, h))$ such that $R_{\bar{h}}=g(\bar{h})$, or $R_{2 / \sqrt{3}}>g(2 / \sqrt{3})=1 / \sqrt{6}$. In the first case we replace $\mathscr{C}(\mathscr{B})$ with $R C C(\bar{h})$ and in the second with $R C C(2 / \sqrt{3})$. These satisfy the two alternatives of the proposition.

Proposition A2. Any facecone $\mathscr{C}(\mathscr{F})$ of height $h \in[1,2 / \sqrt{3}]$ is replaceable by either

(1) an $R C C$ of height $h$ and radius $R \geq \sqrt{\frac{3}{2}-h^{2}}$; or

(2) a regular, vertex-free, order-5 SRCC of height $h$, outer radius $\sqrt{\frac{3}{2}-h^{2}}$, and inner radius $r \geq\left(2-h^{2}\right) / \sqrt{4-h^{2}}$.

Proof. Let $\mathscr{B}$ be the circle of radius $\sqrt{\frac{3}{2}-h^{2}}$ about $M$ in the plane of $\mathscr{F}$. If the solid angle of $\mathscr{C}(\mathscr{F})$ is greater than that of $\mathscr{B}$, then we can replace $\mathscr{C}(\mathscr{F})$ with an $\mathrm{RCC}$ of height $h$ and radius greater than $\sqrt{\frac{3}{2}-h^{2}}$ as in the proof of Proposition A1.

Otherwise, consider $\mathscr{B} \cap \mathscr{F}$. By TLS, this is a shaved circle with at most five chords, one for each edge of $\mathscr{F}$ that lies within $\sqrt{\frac{3}{2}}$ of $A$. By TL4, none of the vertices of $\mathscr{F}$ lies inside $\mathscr{B}$, so $\mathscr{B} \cap \mathscr{F}$ is vertex-free. We can construct a vertex-free order-5 SRCC containing $\mathscr{B} \cap \mathscr{F}$ by shrinking the chords of $\mathscr{B} \cap \mathscr{F}$, i.e., by pulling 
the edges of $\mathscr{F}$ away from $M$. In this way we can construct a vertex-free order-5 SRCC $\mathscr{C}(\mathscr{P})$ of radius $\sqrt{\frac{3}{2}-h^{2}}$ such that $\mathscr{S}$ contains $\mathscr{B} \cap \mathscr{F}$ and $\mathscr{C}(\mathscr{S})$ has the same solid angle as $\mathscr{F}$.

As in the proof of Proposition A1, $\mathscr{F}-\mathscr{S}$ lies outside the circle $\mathscr{B}$ while $\mathscr{S}-\mathscr{F}$ lies inside, so $\mathscr{C}(\mathscr{P})$ has less volume than $\mathscr{C}(\mathscr{F})$. It follows easily from TL4 that $\mathscr{S}$ contains a circle of radius $\left(2-h^{2}\right) / \sqrt{4-h^{2}}$ about $M$, so $\mathscr{S}$ satisfies all the requirements of the proposition other than regularity.

The volume and solid angle of an SRCC of a given height and radius are determined by the number and length of the chords, not by their relative locations. So we may assume that the chords of $\mathscr{S}$ are positioned so that the angles between them are equal. There is a unique inner radius $r \geq\left(2-h^{2}\right) / \sqrt{4-h^{2}}$ such that the regular order-5 SRCC $\hat{\mathscr{P}}$ of height $h$, outer radius $\sqrt{\frac{3}{2}-h^{2}}$, and inner radius $r$ has the same solid angle as $\mathscr{C}(\mathscr{S})$. We may assume that the chords of $\hat{\mathscr{S}}$ are positioned so as to be parallel to the chords of $\mathscr{S}$.

Now $\widehat{\mathscr{S}}-\mathscr{S}$ and $\mathscr{S}-\hat{\mathscr{S}}$ are each unions of infinitesimal chordal bands like those described in TL6. The bands of $\hat{\mathscr{S}}-\mathscr{S}$ all have distance at most $r$ from $M$ while those of $\mathscr{S}-\widehat{\mathscr{S}}$ have distance at least $r$ from $M$. Therefore TL6 implies that $\mathscr{C}(\hat{\mathscr{S}}-\mathscr{P})$ has less volume than $\mathscr{C}(\mathscr{S}-\hat{\mathscr{S}})$.

Proposition A3. Any regular, vertex-free, order-5 SRCC $\mathscr{C}(\mathscr{S})$ of height $h \in[1,2 / \sqrt{3}]$, outer radius $R \geq \sqrt{\frac{3}{2}-h^{2}}$, and inner radius $r \geq\left(2-h^{2}\right) / \sqrt{4-h^{2}}$ is replaceable by either

(1) a regular, vertex-free, order-5SRCC of height $\bar{h} \in(1, h]$, outer radius $\sqrt{\frac{3}{2}-\bar{h}^{2}}$, and inner radius $\left(2-\bar{h}^{2}\right) / \sqrt{4-\bar{h}^{2}} ;$ or

(2) a regular, vertex-free, order-5 SRCC of height 1 , outer radius $1 / \sqrt{2}$, and inner radius $r \in(1 / \sqrt{3}, 1 / \sqrt{2}]$; or

(3) an $R C C$ of height 1 and radius $R \geq 1 / \sqrt{2}$.

Proof. First we show that we can reduce to the case $R=\sqrt{\frac{3}{2}-h^{2}}$. If $R>\sqrt{\frac{3}{2}-h^{2}}$, then let $\bar{R}$ be the smallest element of $\left[\sqrt{\frac{3}{2}-h^{2}}, R\right)$ such that the RCC of height $h$ and radius $\bar{R}$ has solid angle at least that of $\mathscr{C}(\mathscr{S})$. Select $\bar{r} \leq \bar{R}$ so that the regular, vertex-free, order-5 SRCC $\mathscr{C}(\mathscr{S})$ of height $h$, outer radius $\bar{R}$, and inner radius $\bar{r}$ has the same solid angle as $\mathscr{C}(\mathscr{S})$. (Clearly, $R>\bar{R} \geq \bar{r}>r$.) Then $\mathscr{P}-\mathscr{S}$ lies inside the circle of radius $\bar{R}$ about $M$ while $\mathscr{S}-\mathscr{P}$ lies outside. So TL3 allows us to deduce that $\mathscr{C}(\mathscr{S})$ is replaceable by $\mathscr{C}(\mathscr{P})$. Thus we arrive either at $\bar{R}=\sqrt{\frac{3}{2}-h^{2}}$ or $\mathscr{C}(\hat{\mathscr{P}})$ being an RCC of radius greater than $\sqrt{\frac{3}{2}-h^{2}}$. In the latter case let

$$
\bar{h}=\max \left(1, h \sqrt{\frac{3}{2\left(h^{2}+\bar{R}^{2}\right)}}\right)
$$

and consider the RCC $\mathscr{C}(\overline{\mathscr{S}})$ of height $\bar{h}$ that is similar to $\mathscr{C}(\hat{\mathscr{S}})$. It is easy to check 
that $\bar{h} \leq h$ so that $\mathscr{C}(\overline{\mathscr{P}})$ replaces $\mathscr{C}(\hat{\mathscr{S}})$. Now $\mathscr{C}(\overline{\mathscr{P}})$ is either an RCC of height 1 and radius greater than $1 / \sqrt{2}$ (conclusion (3)), or it has $\bar{h} \geq 1$ and radius $\sqrt{\frac{3}{2}-\bar{h}^{2}}$. Thus we can assume $R=\sqrt{\frac{3}{2}-h^{2}}$.

If $h=1$ we have conclusion (2), so assume $h>1$. If $r=\left(2-h^{2}\right) / \sqrt{4-h^{2}}$ we have conclusion (1), so assume $r>\left(2-h^{2}\right) / \sqrt{4-h^{2}}$. Define a set of coordinates such that $A=(0,0,0)$ and $M=(h, 0,0)$. For any $\bar{h} \in[1, h]$, define the regular, vertex-free, order-5 shaved circle $\mathscr{S}(\bar{h})$ to be the image of $\mathscr{S}$ under the linear transformation

$$
f_{\bar{h}}(x, y, z)=\left(\sqrt{\frac{3-2 \bar{h}^{2}}{3-2 h^{2}}} x, \sqrt{\frac{3-2 \bar{h}^{2}}{3-2 h^{2}}} y, \frac{\bar{h}}{h} z\right)
$$

We easily see that $\mathscr{C}(\bar{h})=\mathscr{C}(\mathscr{S}(\bar{h}))$ has height $\bar{h}$ and outer radius $\sqrt{\frac{3}{2}-\bar{h}^{2}}$.

TL7 establishes that $\mathscr{C}(\bar{h})$ has solid angle at least that of $\mathscr{C}(\mathscr{P})$, and that the volume-to-solid-angle ratio of $\mathscr{C}(\bar{h})$ is at most that of $\mathscr{C}(\mathscr{P})$. Construct an SRCC $\hat{\mathscr{C}}(\bar{h})$ by reducing the inner radius of $\mathscr{C}(\bar{h})$ until the solid angle is equal to that of $\mathscr{C}(\mathscr{S})$. By TL8 this lowers the volume-to-solid-angle ratio even further, so that the volume of $\hat{\mathscr{C}}(\bar{h})$ is no greater than that of $\mathscr{C}(\mathscr{S})$.

If the inner radius of $\mathscr{\mathscr { C }}(1)$ is greater than $1 / \sqrt{3}$, then we use this SRCC to replace $\mathscr{C}(\mathscr{S})$ and arrive at conclusion (2). If not, then there is some $\bar{h} \in[1, h]$ such that the inner radius of $\hat{\mathscr{C}}(\bar{h})$ is exactly $\left(2-\bar{h}^{2}\right) / \sqrt{4-\bar{h}^{2}}$. Using this SRCC to replace $\mathscr{C}(\mathscr{S})$ gives us conclusion (1).

Proof of Proposition A. Consider a facecone of height $h>2 / \sqrt{3}$. By Proposition A1 we see that either it can be replaced by an SRCC described in (1) of Proposition $A$, or by an $\mathrm{RCC}$ of height $2 / \sqrt{3}$ and radius $R>1 / \sqrt{6}$. In the latter case we can describe the RCC as a regular, vertex-free, order-5 SRCC with inner and outer radius equal and both greater than $1 / \sqrt{6}$. This allows us to apply Proposition A3. The three possibilities of Proposition A3 correspond to (2)-(4) of Proposition A. So Proposition A holds for all facecones of height at least $2 / \sqrt{3}$.

For facecones of height $h \leq 2 / \sqrt{3}$, we can apply Proposition A2. Again an RCC can be described as a regular, vertex-free order- 5 SRCC with equal inner and outer radii. Thus either possibility in Proposition A2 allows us to apply Proposition A3. We conclude as in the previous case.

\section{Proof of Proposition $B$}

A function that is convex down in an interval can be bounded below by the linear function that agrees with it at the endpoints of the interval. A function that is convex up on an interval can be bounded below by a linear function that agrees with it at the left endpoint and has slope equal to its derivative at the left endpoint. 
These are the ideas used to construct $L(\psi)$. This section proves Propositions B1, B2, and B3 from the technical lemmas and uses them to prove Proposition B.

Proposition B1. The function $\beta(\psi)$ is convex down on $\left(0, \hat{\psi}_{1}\right)$.

Proof. From Proposition $\mathrm{A}^{\prime}$ we can calculate

$$
\begin{aligned}
\frac{d \beta}{d h} & =\frac{\pi\left(2-h^{2}\right)\left(8-18 h^{2}+3 h^{4}\right)}{3\left(4-h^{2}\right)^{2}}, \\
\frac{d^{2} \beta}{d h^{2}} & =\frac{2 h\left(-144+148 h^{2}-36 h^{4}+3 h^{6}\right)}{3\left(4-h^{2}\right)^{3}}, \\
\frac{d \psi}{d h} & =\frac{-2 \pi\left(2-h^{2}\right)}{\sqrt{4-h^{2}}}
\end{aligned}
$$

and

$$
\frac{d^{2} \psi}{d h^{2}}=\frac{2 \pi h\left(6-h^{2}\right)}{\left(4-h^{2}\right)^{3 / 2}}
$$

Now

$$
\frac{d^{2} \beta}{d h^{2}} \frac{d \psi}{d h}-\frac{d \beta}{d h} \frac{d^{2} \psi}{d h^{2}}=\frac{2 \pi^{2} h\left(2-h^{2}\right)^{2}\left(40-10 h^{2}+h^{4}\right)}{\left(4-h^{2}\right)^{7 / 2}}
$$

This clearly has the same sign as $40-10 h^{2}+h^{4}$. Since $h \leq \sqrt{2}$ on this interval we have

$$
40-10 h^{2}+h^{4} \geq 40-10 h^{2} \geq 40-20>0 .
$$

Since $d \psi / d h \leq 0$, we can complete the proof by quoting TL2.

Proposition B2. The function $\beta(\psi)$ is convex down on $\left(\hat{\psi}_{1}, \hat{\psi}_{2}\right)$.

Proof. Let

$$
\gamma(h)=\pi-5 \arctan \left(\frac{\sqrt{4-3 h^{2}}}{\sqrt{2}\left(2-h^{2}\right)}\right) .
$$

The interval in question is $h \in(1,2 / \sqrt{3})$, so it is easy to see that $\sqrt{4-3 h^{2}} \leq 2-h^{2}$, and so $\gamma(h)>0$ throughout. 
From Proposition $A^{\prime}$ we can calculate

$$
\begin{aligned}
\frac{d \psi}{d h} & =\frac{-2 \sqrt{2}}{\sqrt{3}\left(4-h^{2}\right)}\left(5 \sqrt{2} \sqrt{4-3 h^{2}}+\left(4-h^{2}\right) \gamma(h)\right), \\
\frac{d^{2} \psi}{d h^{2}} & =\frac{20 h\left(20-13 h^{2}-3 h^{4}\right)}{\sqrt{3} \sqrt{4-3 h^{2}}\left(3-2 h^{2}\right)\left(4-h^{2}\right)^{2}}, \\
\frac{d \beta}{d h} & =\frac{1}{6\left(4-h^{2}\right)^{2}}\left(5 \sqrt{2} \sqrt{4-3 h^{2}}\left(8-18 h^{2}+3 h^{4}\right)+3\left(1-2 h^{2}\right)\left(4-h^{2}\right)^{2} \gamma(h)\right),
\end{aligned}
$$

and

$$
\frac{d^{2} \beta}{d h^{2}}=\frac{-2 h\left(5 \sqrt{2}\left(432-836 h^{2}+522 h^{4}-111 h^{6}+9 h^{8}\right)\right)}{3 \sqrt{4-3 h^{2}}\left(3-2 h^{2}\right)\left(4-h^{2}\right)^{3}}-2 h \gamma(h)
$$

Now

$$
\frac{d^{2} \beta}{d h^{2}} \frac{d \psi}{d h}-\frac{d \beta}{d h} \frac{d^{2} \psi}{d h^{2}}=\frac{2 h}{\sqrt{4-3 h^{2}}\left(3-2 h^{2}\right)\left(4-h^{2}\right)^{4}}\left(\alpha_{0}(h)+\alpha_{1}(h) \gamma(h)+\alpha_{2}(h) \gamma(h)^{2}\right)
$$

where

$$
\begin{aligned}
& \alpha_{0}(h)=\frac{25 \sqrt{2} \sqrt{4-3 h^{2}}\left(1568-2880 h^{2}+1818 h^{4}-459 h^{6}+45 h^{8}\right)}{\sqrt{27}}, \\
& \alpha_{1}(h)=\frac{5\left(4-h^{2}\right)\left(3-2 h^{2}\right)\left(1264-1512 h^{2}+519 h^{4}-63 h^{6}\right)}{\sqrt{27}}, \\
& \alpha_{2}(h)=\frac{6 \sqrt{2}\left(4-h^{2}\right)^{4}\left(3-2 h^{2}\right) \sqrt{4-3 h^{2}}}{\sqrt{27}} .
\end{aligned}
$$

Since $d \psi / d h \leq 0$ and $\gamma(h) \geq 0$, we need only show that all $\alpha_{i}(h) \geq 0$ for $h \in[1,2 / \sqrt{3}]$ to be able to complete the proof by quoting TL2.

If we let $w=4-h^{2}, x=4-3 h^{2}, y=h^{2}-1$, and $z=3-2 h^{2}$, then it is easily checked that $w, x, y, z \geq 0$, and

$$
\begin{aligned}
& \alpha_{0}(h)=25 \sqrt{2 x}\left(14+9 x+2 y^{2}+69 x^{2}+83 x y^{2}+5 x^{2} y^{2}\right) \geq 0 \\
& \alpha_{1}(h)=5 w z\left(21+153 x+34 x^{2}+3 y^{2}+21 x y^{2}\right) \geq 0
\end{aligned}
$$


and

$$
\alpha_{2}(h)=6 \sqrt{2 x} w^{4} z \geq 0
$$

Proposition B3. The function $\beta(\psi)$ is convex up on $\left(\hat{\psi}_{2}, 2 \pi\right)$.

Proof. From TL9 we see that $\beta(\psi)$ is convex up on $\left(\hat{\psi}_{2}, \hat{\psi}_{3}\right)$, and that the left-hand derivative of $\beta$ at $\hat{\psi}_{3}$ is $\sqrt{\frac{3}{8}}$.

From Proposition $A^{\prime}$ we can easily calculate

$$
\beta^{\prime}(\psi)=\frac{d \beta_{R} / d R}{d \psi_{R} / d R}=\frac{1}{3}\left(R^{2}+1\right)^{3 / 2}
$$

for $\psi \in\left(\hat{\psi}_{3}, 2 \pi\right)$. From this and the geometrically obvious fact that $\psi_{R}$ is increasing with $R$, we see that $\beta^{\prime}(\psi)$ is increasing on $\left(\hat{\psi}_{3}, 2 \pi\right)$. In addition, we see that the right-hand derivative of $\beta$ at $\hat{\psi}_{3}$ is $\sqrt{\frac{3}{8}}$, which equals the left-hand derivative.

Proof of Proposition $B$. It is easy to check that $L(\psi)$ agrees with $\beta(\psi)$ at $0, \hat{\psi}_{1}$, and $\hat{\psi}_{2}$. Because it is linear on each of the intervals $\left(0, \hat{\psi}_{1}\right)$ and $\left(\hat{\psi}_{1}, \hat{\psi}_{2}\right)$, the convexity results of Propositions B1 and B2 allow us to conclude that $\beta(\psi) \geq L(\psi)$ for all $\psi \leq \hat{\psi}_{2}$. Since $L(\psi)=\beta(\psi)$ on $\left[\hat{\psi}_{2}, \hat{\psi}_{3}\right]$, we need only check what happens on $\left[\hat{\psi}_{3}, 2 \pi\right)$. Here $L(\psi)$ is linear and its graph is tangent to that of $\beta(\psi)$ at $\hat{\psi}_{3}$. So the conclusion follows from the convexity established by Proposition B3.

\section{Proof of Proposition C}

Once the convexity of $L(\psi)$ is known, it is intuitively clear that the sum $\sum L\left(\psi_{i}\right)$ is minimized by having all solid angles greater than $\hat{\psi}_{2}$ be equal and eliminating all but at most one of the solid angles less than $\hat{\psi}_{2}$. It is also clear that increasing the number of solid angles above $\hat{\psi}_{2}$ allows each of them to be smaller, closer to the ideal solid angle $\hat{\psi}_{2}$. The only suspense in the calculation is whether the minimal configuration will be 13 solid angles of measure $4 \pi / 13$, or 13 of measure $\hat{\psi}_{2}$ and one of $4 \pi-13 \hat{\psi}_{2}$. Evaluation shows that the former is minimal.

Proposition C1. $L(\psi)$ is convex down on $\left(0, \hat{\psi}_{2}\right)$.

Proof. On $\left(0, \hat{\psi}_{1}\right), L(\psi)$ is linear with slope $\beta\left(\hat{\psi}_{1}\right) / \hat{\psi}_{1}$. This can be evaluated (using the formulas of Proposition $A^{\prime}$ ) to yield

$$
L^{\prime}(\psi)=\frac{1}{6 \sqrt{3(3-2 \sqrt{2})}} \sim 0.5608
$$

on $\left(0, \hat{\psi}_{1}\right)$. 
Similarly, we can deduce

$$
\begin{aligned}
L^{\prime}(\psi) & =\frac{\beta\left(\hat{\psi}_{2}\right)-\beta\left(\hat{\psi}_{1}\right)}{\hat{\psi}_{2}-\hat{\psi}_{1}} \\
& =\frac{5 \sqrt{6}+(3 \sqrt{3}-2) \pi-15 \sqrt{3} \arctan (1 / \sqrt{2})}{(24 \sqrt{6}-36 \sqrt{2}-30 \sqrt{3}) \pi+180 \sqrt{2} \arctan (1 / \sqrt{2})} \\
& \sim 0.3465
\end{aligned}
$$

for $\psi \in\left(\hat{\psi}_{1}, \hat{\psi}_{2}\right)$. Therefore $L(\psi)$ is convex down on $\left(0, \hat{\psi}_{2}\right)$.

Proposition C2. Let $k \leq 13$ be a positive integer, let $\psi_{0}, \ldots, \psi_{N}$ be as in $C$, and define

$$
V_{k}(\psi)=L(\psi)+k L\left(\frac{4 \pi-\psi}{k}\right)
$$

for all $\psi \in\left[0, \min \left(\hat{\psi}_{2}, 4 \pi-k \hat{\psi}_{2}\right)\right)$. Then

$$
\sum_{i=0}^{N} L\left(\psi_{i}\right) \geq V_{K}(\psi)
$$

for some $K \leq \min (N, 13)$ and $\psi \in\left[0, \min \left(\hat{\psi}_{2}, 4 \pi-K \hat{\psi}_{2}\right)\right)$.

Proof. Suppose $0<\psi_{0}<\psi_{1}<\hat{\psi}_{2}$. Since $L(\psi)$ is convex down on $\left(0, \hat{\psi}_{2}\right), \psi_{0}$ and $\psi_{1}$ are replaceable by $\psi_{0}-t$ and $\psi_{1}+t$, where $t=\min \left(\psi_{0}, \hat{\psi}_{2}-\psi_{1}\right)$. Repeated use of this technique, along with the possible adding or deleting of zeros from $\left(\psi_{0}, \ldots, \psi_{N}\right)$, leads us to a solid angle decomposition that has precisely one element (say $\psi_{0}$ ) in the interval $\left[0, \hat{\psi}_{2}\right)$ and $K$ elements in $\left[\hat{\psi}_{2}, 2 \pi\right.$ ). Since $14 \hat{\psi}_{2}>4 \pi$ we see that $K$ is at most 13 .

Similarly, Proposition B3 and the definition of $L$ allow us to deduce that $L(\psi)$ is convex up on $\left(\hat{\psi}_{2}, 2 \pi\right)$. Therefore we can replace $\psi_{1}, \ldots, \psi_{K}$ with $K$ identical angles of size $\left(4 \pi-\psi_{0}\right) / K$. Now

$$
\sum_{i=0}^{K} L\left(\psi_{i}\right)=V_{K}\left(\psi_{0}\right)
$$

Proposition C3. If $K<13$, then $V_{K}(\psi) \geq V_{K+1}(0)$.

Proof. Define

$$
g(\psi)= \begin{cases}\frac{\beta\left(\hat{\psi}_{2}\right)}{\hat{\psi}_{2}} \psi & \text { for } \quad \psi \in\left[0, \hat{\psi}_{2}\right], \\ L(\psi) & \text { for } \quad \psi \in\left[\hat{\psi}_{2}, 2 \pi\right)\end{cases}
$$


Because $g$ is linear on $\left[0, \hat{\psi}_{2}\right]$ and agrees with $L$ at the endpoints, the fact that $L$ is convex down on this interval allows us to deduce that $g(\psi) \leq L(\psi)$ on $\left[0, \hat{\psi}_{2}\right]$, and thus $g(\psi) \leq L(\psi)$ everywhere.

We see from Proposition $A^{\prime}$ that

$$
g^{\prime}(\psi)=\frac{\beta\left(\hat{\psi}_{2}\right)}{\hat{\psi}_{2}}=\frac{5 \sqrt{2}+3 \pi-15 \arctan (1 / \sqrt{2})}{6(1-2 \sqrt{6}) \pi+60 \sqrt{6} \arctan (1 / \sqrt{2})} \sim 0.4282
$$

on $\left(0, \hat{\psi}_{2}\right)$. We can also use Proposition $\mathrm{A}^{\prime}$ and TL9 to see that

$$
g^{\prime}(\psi)=g^{\prime}\left(\psi_{r}\right)=\beta^{\prime}\left(\psi_{r}\right)=\frac{1+r^{2}}{\sqrt{6}}
$$

for $\psi=\psi_{r} \in\left(\hat{\psi}_{2}, \hat{\psi}_{3}\right)$ (i.e., $\left.r \in(1 / \sqrt{3}, 1 / \sqrt{2})\right)$. Thus $g^{\prime}(\psi)$ increases on $\left(\hat{\psi}_{2}, \hat{\psi}_{3}\right)$ from $\sqrt{8 / 27} \sim 0.5443$ to $\sqrt{3 / 8} \sim 0.6124$, and remains constant thereafter. Thus we see that $g(\psi)$ is convex up on $(0,2 \pi)$ and

$$
\begin{aligned}
V_{K}(\psi) & =L(\psi)+K L\left(\frac{4 \pi-\psi}{K}\right) \\
& \geq g(\psi)+K g\left(\frac{4 \pi-\psi}{K}\right) \\
& \geq(K+1) g\left(\frac{4 \pi}{K+1}\right) .
\end{aligned}
$$

Because $K<13$, it follows that $4 \pi /(K+1)>\hat{\psi}_{2}$, and therefore

$$
(K+1) g\left(\frac{4 \pi}{K+1}\right)=(K+1) L\left(\frac{4 \pi}{K+1}\right)=V_{K+1}(0) .
$$

Proposition C4. The function $V_{13}(\psi)$ is increasing.

Proof. By definition

$$
V_{13}^{\prime}(\psi)=L^{\prime}(\psi)-L^{\prime}\left(\frac{4 \pi-\psi}{13}\right)=L^{\prime}(\psi)-\beta^{\prime}\left(\frac{4 \pi-\psi}{13}\right)
$$

The function $V_{13}(\psi)$ is defined on $\psi \in\left[0,4 \pi-13 \hat{\psi}_{2} \sim 0.3156\right] \subset\left[0, \hat{\psi}_{1}\right]$. We have previously (during the proof of Proposition C2) calculated

$$
L^{\prime}(\psi)=\frac{1}{6 \sqrt{3}(3-2 \sqrt{2})} \sim 0.5608
$$


on this interval. Since $(4 \pi-\psi) / 13 \in\left[\hat{\psi}_{2}, 4 \pi / 13\right] \subset\left[\hat{\psi}_{2}, \hat{\psi}_{3}\right]$, TL9 shows

$$
\beta^{\prime}\left(\frac{4 \pi-\psi}{13}\right)=\frac{1+r^{2}}{\sqrt{6}}
$$

The value of $r$ corresponding to $4 \pi / 13$ is less than 0.59 , so it follows that

$$
\beta^{\prime}\left(\frac{4 \pi-\psi}{13}\right)<0.5504
$$

Therefore $V_{13}^{\prime}(\psi)>0$ throughout the interval.

Proof of Proposition $C$. The propositions above establish that

$$
\sum_{i=0}^{M} L\left(\psi_{i}\right) \geq V_{K}(\psi) \geq V_{13}(0)=13 L\left(\frac{4 \pi}{13}\right)
$$

\section{Technical Lemmas}

The section contains proofs of Technical Lemmas TL1-TL8.

TL1. Let $0<h, r ; r \leq R$; and let $n$ be a positive integer such that

$$
n \arctan \sqrt{\frac{R^{2}-r^{2}}{r^{2}}} \leq \pi
$$

Then the vertex-free regular SRCC of order $n$, height $h$, inner radius $r$, and outer radius $R$ has volume

$$
\beta_{n}(h, r, R)=\frac{h}{3}\left(n r \sqrt{R^{2}-r^{2}}+R^{2}\left(\pi-n \arctan \sqrt{\frac{R^{2}-r^{2}}{r^{2}}}\right)\right)
$$

and solid angle

$$
\begin{aligned}
\psi_{n}(h, r, R)= & 2\left(\pi-n \arctan \left(\sqrt{\frac{h^{2}}{R^{2}+h^{2}}} \sqrt{\frac{R^{2}-r^{2}}{r^{2}}}\right)\right) \\
& -2 \sqrt{\frac{h^{2}}{R^{2}+h^{2}}}\left(\pi-n \arctan \sqrt{\frac{R^{2}-r^{2}}{r^{2}}}\right) .
\end{aligned}
$$

Proof. The face of the SRCC consists of $2 n$ identical right triangles with legs of length $r$ and $\sqrt{R^{2}-r^{2}}$, together with $n$ circular sectors of radius $R$, such that the 
total angle at $M$ adds up to $2 \pi$. The total area of the triangles is $n r \sqrt{R^{2}-r^{2}}$ and their angles at $M$ add up to

$$
2 n \arctan \sqrt{\frac{R^{2}-r^{2}}{r^{2}}}
$$

The total area of the circular sectors is therefore

$$
\pi R^{2} \frac{2 \pi-2 n \arctan \sqrt{\left(R^{2}-r^{2}\right) / r^{2}}}{2 \pi}=R^{2}\left(\pi-n \arctan \sqrt{\frac{R^{2}-r^{2}}{r^{2}}}\right)
$$

The volume calculation follows immediately.

The solid angle of the cone over each of the $2 n$ triangles is the surface area of the projection of the triangle onto a unit sphere about the apex. The surface area of a spherical triangle is the sum of the angles on the sphere minus $\pi$. From this we calculate that each of these triangles has solid angle

$$
\begin{aligned}
& -\frac{\pi}{2}+\arctan \sqrt{\frac{R^{2}-r^{2}}{r^{2}}}+\arctan \left(\frac{r \sqrt{R^{2}+h^{2}}}{h \sqrt{R^{2}-r^{2}}}\right) \\
& \quad=\arctan \sqrt{\frac{R^{2}-r^{2}}{r^{2}}}-\arctan \left(\sqrt{\frac{h^{2}}{R^{2}+h^{2}}} \sqrt{\frac{R^{2}-r^{2}}{r^{2}}}\right) .
\end{aligned}
$$

The solid angle of an RCC of height $h$ and radius $R$ is the surface area of a unit spherical cap of height $h / \sqrt{R^{2}+h^{2}}$, or

$$
2 \pi\left(1-\frac{h}{\sqrt{R^{2}+h^{2}}}\right)
$$

The solid angle formula follows quickly.

TL2. Suppose that $\beta(\psi)$ is described in the interval $(a, b)$ in terms of the parameter t. Then $\beta(\psi)$ is convex up on $(a, b)$ if

$$
\frac{d^{2} \beta}{d t^{2}}\left(\frac{d \psi}{d t}\right)^{2} \geq \frac{d \beta}{d t} \frac{d^{2} \psi}{d t^{2}} \frac{d \psi}{d t}
$$

and convex down if

$$
\frac{d^{2} \beta}{d t^{2}}\left(\frac{d \psi}{d t}\right)^{2} \leq \frac{d \beta}{d t} \frac{d^{2} \psi}{d t^{2}} \frac{d \psi}{d t}
$$

for all $t$ such that $\psi(t) \in(a, b)$. 
Proof. A simple application of the chain rule shows that

$$
\left(\frac{d \psi}{d t}\right)^{3} \frac{d^{2} \beta}{d \psi^{2}}=\frac{d^{2} \beta}{d t^{2}} \frac{d \psi}{d t}-\frac{d \beta}{d t} \frac{d^{2} \dot{\psi}}{d t^{2}}
$$

Therefore $d^{2} \beta / d \psi^{2}$ has the same sign as

$$
\frac{d \psi}{d t}\left(\frac{d^{2} \beta}{d t^{2}} \frac{d \psi}{d t}-\frac{d \beta}{d t} \frac{d^{2} \psi}{d t^{2}}\right)
$$

and the result follows.

TL3. Let $\mathscr{P}$ be a plane and let $A$ be a point of distance $h$ from $\mathscr{P}$. Let $M$ be the perpendicular projection of $A$ into $\mathscr{P}$. Define a set of rectangular coordinates $(x, y)$ in $\mathscr{P}$ such that $M=(0,0)$. Let $\mathscr{R}(x, y)$ be the infinitesimal rectangle $[x, x+d x] \times$ $[y, y+d y]$. Then the cone $\mathscr{C}(x, y)$ with base $\mathscr{R}(x, y)$ and apex $A$ has volume

$$
V(x, y)=\frac{h}{3} d x d y
$$

and solid angle

$$
\psi(x, y)=\frac{h d x d y}{\left(h^{2}+x^{2}+y^{2}\right)^{3 / 2}}
$$

Moreover, the ratio

$$
\frac{V(x, y)}{\psi(x, y)}=\frac{1}{3}\left(h^{2}+x^{2}+y^{2}\right)^{3 / 2}=\frac{\rho^{3}}{3},
$$

where $\rho=\rho(x, y)$ is the distance from $(x, y)$ to $A$.

Proof. The volume formula is elementary, and the solid angle formula follows quickly from the ratio formula.

Consider the ball $\mathscr{B}$ of radius $\rho$ about $A$. Let $\overline{\mathscr{R}}(x, y)$ be the projection from $A$ of $\mathscr{R}(x, y)$ onto the surface of $\mathscr{B}$. Let $\overline{\mathscr{C}}(x, y)$ be the set of all points between $A$ and $\overline{\mathscr{R}}(x, y)$ (i.e., the "cone" over $\overline{\mathscr{R}}(x, y))$.

Clearly, $\mathscr{C}(x, y)$ has the same solid angle at $A$ as $\overline{\mathscr{C}}(x, y)$, and the difference in volumes is a third-degree infinitesimal, which can be ignored. The volume-to-solidangle ratio of $\overline{\mathscr{C}}(x, y)$ is the same as that of $\mathscr{B}$, i.e., $\rho^{3} / 3$.

TL4. Let $\mathscr{F}$ be a face of a Voronoi polyhedron $\mathscr{V}$ with center $A$. Let $h$ be the distance from $\mathscr{F}$ to $A$, and suppose $h \leq \sqrt{2}$. Then no vertex of $\mathscr{V}$ is closer than $\sqrt{\frac{3}{2}}$ to $A$ and no edge of $\mathscr{F}$ is closer than $2 / \sqrt{4-h^{2}}$ to $A$. 
Proof. By definition a vertex of $\mathscr{V}$ is the intersection of $\mathscr{V}$ with three or more of its neighbors, and the centers of these polyhedra lie on a sphere centered at the vertex. The smallest sphere that contains a set of four or more points of minimum distance 2 is the sphere superscribing a regular tetrahedron of side 2 . The radius of this sphere is $\sqrt{\frac{3}{2}}$. Thus any vertex of the Voronoi polyhedron about $A$ is at least $\sqrt{\frac{3}{2}}$ from $A$.

Similarly, an edge of $\mathscr{V}$ is the intersection of $\mathscr{V}$ with two or more neighbors whose centers all lie in a circle with $A$. The radius of the circle is the distance from each of the centers to the line containing the edge. Let $B$ be the center of the polyhedron that meets $\mathscr{V}$ at $\mathscr{F}$. Let $C$ be a third center on the circle. By assumption $A B=2 h$ while $A C, B C \geq 2$. It is easy to see that the smallest circle containing such a configuration has radius $2 / \sqrt{4-h^{2}}$.

TL5. Let $\mathscr{F}$ be a face of a Voronoi polyhedron $\mathscr{V}$ with center A. At most five edges of $\mathscr{F}$ are less than $\sqrt{\frac{3}{2}}$ from $A$.

Proof. Let $B$ be the center of the Voronoi polyhedron that intersects $\mathscr{V}$ in the face $\mathscr{F}$, and let $M$ be the midpoint of $\overline{A B}$. Let $h=M A=M B$, and note that we can assume that $1 \leq h<\sqrt{\frac{3}{2}}$.

Next we observe that $M \in \mathscr{F}$. If not, then it is part of some other Voronoi polyhedron with center $C$, so $M C<h$. Now

$$
\min (\angle C M A, \angle C M B) \leq \frac{\pi}{2}
$$

so the law of cosines and the fact that $A C, B C \geq 2$ forces $h>\sqrt{2}$, contradicting our assumption.

Let $\mathscr{E}_{1}, \ldots, \mathscr{E}_{n}$ be the edges of $\mathscr{F}$ that are less than $\sqrt{\frac{3}{2}}$ from $A$. Let $E_{1}, \ldots, E_{n}$ be the centers of Voronoi polyhedra $\mathscr{V}_{1}, \ldots, \mathscr{V}_{n}$ such that $\mathscr{V}_{i} \cap \mathscr{F}=\mathscr{E}_{i}$. The convexity of Voronoi polyhedra ensures that all the $\mathscr{V}_{i}$ (and hence all the $E_{i}$ ) are distinct. Let $\mathscr{P}_{i}$ be the plane containing $A, B$, and $E_{i}$.

If $n \geq 6$, then there are $i$ and $j$ such that the angle between $\mathscr{P}_{i}$ and $\mathscr{P}_{j}$ is $\pi / 3$ or less. We complete the proof by showing that this forces $E_{i} E_{j}<2$, an impossibility.

Let $\mathscr{C}_{i}$ be the circle containing $\left\{A, B, E_{i}\right\}$, let $C_{i}$ be its center and let $R_{i}$ be its radius. By assumption, $R_{i}<\sqrt{\frac{3}{2}}$ since it is the distance from $A$ to $\mathscr{E}_{i}$. Let $\hat{\mathscr{C}}_{i}$ be the circle of radius $\sqrt{\frac{3}{2}}$ in $\mathscr{P}_{i}$ that contains $\{A, B\}$ and whose center $\hat{C}_{i}$ lies on $M C_{i}$. Let $\hat{E}_{i}=\hat{\mathscr{C}}_{i} \cap M E_{i}$ and let $\vec{E}_{i}$ be the projection of $\hat{E}_{i}$ onto $M C_{i}$. Let $\varphi_{i}$ be the angle $\angle \hat{E}_{i} \hat{C}_{i} \bar{E}_{i}$. Let $\mu_{i}=M \bar{E}_{i}$. This notation is shown in Fig. 3. Define analogous notation for the subscript $j$.

Let $l_{i}=M E_{i}$ and $\hat{l}_{i}=M \hat{E}_{i}$. It is easy to see that $\hat{l}_{i}>l_{i}$. Let $\eta=\angle E_{i} M E_{j}=$ $\angle \hat{E}_{i} M \hat{E}_{j}$. The Law of Cosines says that

$$
E_{i} E_{j}=l_{i}^{2}+l_{j}^{2}-2 l_{i} l_{j} \cos \eta
$$




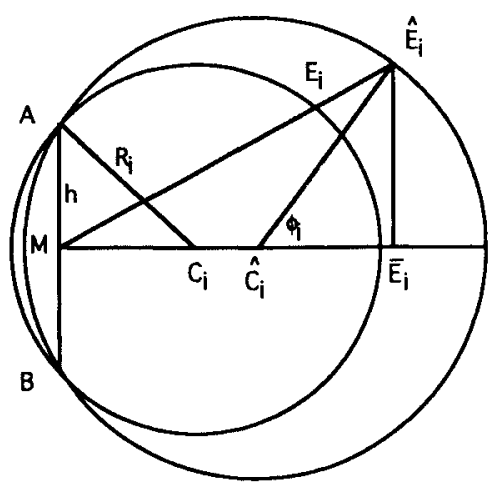

Fig. 3.

Differentiation shows that the right-hand side increases with $l_{i}$ iff $l_{i}>l_{j} \cos \eta$. It is easy to see that this is always true: If $l_{i} \leq l_{j} \cos \eta$, then $\angle M E_{i} E_{j}$ is at least $\pi / 2$ and so

$$
l_{j}^{2} \geq l_{i}^{2}+\left(E_{i} E_{j}\right)^{2}>4
$$

However,

$$
l_{j}=M E_{j}<M C_{j}+C_{j} E_{j}=\sqrt{R_{j}^{2}-h^{2}}+R_{j}<\frac{1+\sqrt{3}}{\sqrt{2}}<2 .
$$

Therefore

$$
\left(E_{i} E_{j}\right)^{2}=l_{i}^{2}+l_{j}^{2}-2 l_{i} l_{j} \cos \eta<\hat{l}_{i}^{2}+\hat{l}_{j}^{2}-2 \hat{l} \hat{l}_{j} \cos \eta=\left(\hat{E}_{i} \hat{E}_{j}\right)^{2} .
$$

Consequently it will suffice to prove that $\hat{E}_{i} \hat{E}_{j} \leq 2$. We observe that

$$
\begin{aligned}
\left(\hat{E}_{i} \hat{E}_{j}\right)^{2} & \leq\left(\widehat{E}_{i} \bar{E}_{i}+\hat{E}_{j} \bar{E}_{j}\right)^{2}+\left(\widetilde{E}_{i} \bar{E}_{j}\right)^{2} \\
& \leq \frac{3}{2}\left(\sin \varphi_{i}+\sin \varphi_{j}\right)^{2}+\mu_{i}^{2}+\mu_{j}^{2}-2 \mu_{i} \mu_{j} \cos \theta \\
& \leq \frac{3}{2}\left(\sin \varphi_{i}+\sin \varphi_{j}\right)^{2}+\mu_{i}^{2}+\mu_{j}^{2}-\mu_{i} \mu_{j}
\end{aligned}
$$

Substituting

$$
\mu_{i}=\sqrt{\frac{3}{2}-h^{2}}+\sqrt{\frac{3}{2}} \cos \varphi_{i}
$$

yields

$$
\left(\hat{E}_{i} \hat{E}_{j}\right)^{2} \leq \frac{7}{2}+3 \sin \varphi_{i} \sin \varphi_{j}-\frac{3}{2} \cos \varphi_{i} \cos \varphi_{j}+\frac{\sqrt{3}}{2}\left(\cos \varphi_{i}+\cos \varphi_{j}\right)
$$


Differentiating the right-hand side with respect to $\varphi_{i}$ gives

$$
3 \sin \varphi_{j} \cos \varphi_{i}+\frac{3}{2}\left(\cos \varphi_{j}-\frac{1}{\sqrt{3}}\right) \sin \varphi_{i}
$$

which we claim is always positive because $\cos \varphi_{j}>1 / \sqrt{3}$. To see this observe that $\varphi_{j}$ is maximized when $h$ and $A \hat{E}_{j}$ are as small as possible. Using $h \geq 1$ and $A \widehat{E}_{j} \geq A E_{j} \geq 2$ gives

$$
\begin{gathered}
\varphi_{j} \leq \pi-3 \arcsin \left(\sqrt{\frac{2}{3}}\right)=\arccos \left(-\frac{5}{\sqrt{27}}\right), \\
\cos \varphi_{j} \geq \frac{5}{\sqrt{27}}>\frac{1}{\sqrt{3}} .
\end{gathered}
$$

Therefore the bound on $\left(\hat{E}_{i} \hat{E}_{j}\right)^{2}$ is maximized when $\varphi_{i}$ and $\varphi_{j}$ are as large as possible. Using $\varphi_{i}, \varphi_{j} \leq \arccos (5 / \sqrt{27})$ yields

$$
\left(\hat{E}_{i} \hat{E}_{j}\right)^{2} \leq 4
$$

as desired.

TL6. Let $\mathscr{P}, A, M, h$, and the coordinate system $(x, y)$ be as in TL3. Let $\mathscr{B}$ be a disk of radius $R$ in $\mathscr{P}$ with center $M$. For any $x \in[-R, R]$ let $\mathscr{R}(x)$ be the infinitesimal chordal band

$$
\mathscr{R}(x)=\{(\alpha, \beta) \in \mathscr{B} \mid \alpha \in[x, x+d x]\}
$$

Then the cone with base $\mathscr{R}(x)$ and apex $A$ has volume

$$
V(x)=\frac{2 h}{3} \sqrt{R^{2}-x^{2}} d x
$$

and solid angle

$$
\psi(x)=\frac{2 h}{h^{2}+x^{2}} \sqrt{\frac{R^{2}-x^{2}}{R^{2}+h^{2}}} d x
$$

Moreover, the ratio is given by

$$
\frac{V(x)}{\psi(x)}=\frac{1}{3}\left(h^{2}+x^{2}\right) \sqrt{R^{2}+h^{2}} .
$$


Proof. The volume formula is elementary and the ratio formula is simple division, so we need only establish the solid-angle formula. Applying TL3 gives us

$$
\begin{aligned}
\psi(x) & =\int_{\mathscr{R}(x)} \psi(x, y) \\
& =2 h d x \int_{0}^{\sqrt{R^{2}-x^{2}}} \frac{d y}{\left(h^{2}+x^{2}+y^{2}\right)^{3 / 2}} \\
& =2 h d x\left(\frac{y}{\left(h^{2}+x^{2}\right) \sqrt{h^{2}+x^{2}+y^{2}}}\right)_{0}^{\sqrt{R^{2}-x^{2}}} \\
& =\frac{2 h}{h^{2}+x^{2}} \sqrt{\frac{R^{2}-x^{2}}{R^{2}+h^{2}}} d x .
\end{aligned}
$$

TL7. Let $\mathscr{R}$ be a region in a plane $\mathscr{P}$. Let $h \in\left(1 / \sqrt{2}, \sqrt{\frac{3}{2}}\right)$, and suppose that $\mathscr{R}$ lies inside a disk with center $M$ and radius $R=\sqrt{\frac{3}{2}-h^{2}}$. Let $A$ be a point of distance $h$ from $\mathscr{P}$, and suppose that $M$ is the point of $\mathscr{P}$ closest to $A$. Let $\mathscr{C}$ be the cone over $\mathscr{R}$ with apex $A$.

Choose a set of coordinates such that $A=(0,0,0)$ and $M=(0,0, h)$. For any $\bar{h} \in(1 / \sqrt{2}, h]$, let

$$
f_{\bar{h}}(x, y, z)=\left(\sqrt{\frac{3-2 \bar{h}^{2}}{3-2 h^{2}}} x, \sqrt{\frac{3-2 \bar{h}^{2}}{3-2 h^{2}}} y, \frac{\bar{h}}{h} z\right)
$$

Then $f_{\hat{h}}(\mathscr{C})$ has solid angle at least that of $\mathscr{C}$, and the volume-to-solid-angle ratio of $f_{\bar{h}}(\mathscr{C})$ is at most that of $\mathscr{C}$.

Proof. If $(x, y, h) \in \mathscr{R}$, then

$$
\begin{aligned}
|(x, y, h)|^{2}-\left|f_{h}(x, y, h)\right|^{2} & =\left(x^{2}+y^{2}\right)\left(1-\frac{3-2 \bar{h}^{2}}{3-2 h^{2}}\right)+\left(h^{2}-\bar{h}^{2}\right) \\
& =\left(h^{2}-\bar{h}^{2}\right)\left(1-\frac{x^{2}+y^{2}}{R^{2}}\right) \geq 0
\end{aligned}
$$

Therefore $f_{h}$ moves each point of $\mathscr{R}$ closer to $A$.

Let $V_{\bar{h}}(x, y)$ and $\psi_{\bar{h}}(x, y)$ be the volume and solid angle, respectively, of the image under $f_{\bar{h}}$ of the cone over the infinitesimal rectangle $[x, x+d x] \times$ $[y, y+d y] \times\{h\}$. By TL3 we know that

$$
\psi_{h}(x, y)=\frac{3 V_{\bar{h}}(x, y)}{\left|f_{h}(x, y, h)\right|^{3}} \geq \frac{3 V_{\bar{h}}(x, y)}{|(x, y, h)|^{3}} .
$$


Therefore the solid angle $\Psi_{\bar{h}}$ of $f_{\hat{h}}(\mathscr{C})$ is given by

$$
\Psi_{\tilde{h}}=\int_{\mathscr{R}} \psi_{\bar{h}}(x, y) \geq \int_{\mathscr{R}} \frac{3 V_{h}(x, y)}{|(x, y, h)|^{3}} .
$$

However,

$$
V_{h}(x, y)=\frac{\bar{h}\left(3-2 \bar{h}^{2}\right)}{h\left(3-2 h^{2}\right)} V_{h}(x, y),
$$

so

$$
\Psi_{\bar{h}} \geq \frac{\bar{h}\left(3-2 \bar{h}^{2}\right)}{h\left(3-2 h^{2}\right)} \int_{\mathscr{R}} \frac{3 V_{h}(x, y)}{|(x, y, h)|^{3}}=\frac{\bar{h}\left(3-2 \bar{h}^{2}\right)}{h\left(3-2 h^{2}\right)} \Psi_{h} .
$$

Since $3 x-2 x^{3}$ is a decreasing function for $x>1 / \sqrt{2}$, it follows that $\Psi_{\bar{h}} \geq \Psi_{h}$. Since $f_{\bar{h}}$ is the identity, $\Psi_{h}$ is the solid angle of $\mathscr{C}$.

If $\mathscr{V}_{\bar{h}}$ is the volume of $f_{\hat{h}}(\mathscr{C})$, then

$$
\frac{\mathscr{V}_{\bar{h}}}{\Psi_{\bar{h}}}=\frac{\bar{h}\left(3-2 \bar{h}^{2}\right)}{h\left(3-2 h^{2}\right)} \frac{\mathscr{V}_{h}}{\Psi_{\bar{h}}} \leq \frac{\mathscr{V}_{h}}{\Psi_{h}} .
$$

TL8. Let $\mathscr{C}$ and $\overline{\mathscr{C}}$ be regular order $n S R C C$ s of height $h$, outer radius $R$, and inner radii $r$ and $\bar{r}$, respectively, with $\bar{r} \leq r$. Then the volume-to-solid-angle ratio of $\overline{\mathscr{C}}$ is no greater than that of $\mathscr{C}$.

Proof. We can assume that the chords of the base of $\mathscr{C}$ are equally spaced around the circle. Cut the base of $\mathscr{C}$ into $2 n$ identical central sectors in the following way: Consider the rays from the center $M$ that bisect the $n$ chords, and for each such ray construct the two rays that make an angle of measure $\pi / n$ with it. The volume-to-solid-angle ratio of $\mathscr{C}$ is the same as that of cones over each of these $2 n$ central sectors.

Consider a single central sector of $\mathscr{C}$. Define a set of coordinates on the plane of the base such that $M=(0,0)$ and the midpoint of the chord is $(r, 0)$. Following TL3, the volume and solid angle of the sector are given by

$$
V(r)=\int_{0}^{r} \int_{0}^{\min \left(x \tan (\pi / n) \cdot \sqrt{R^{2}-x^{2}}\right)} \frac{h}{3} d y d x=\int_{0}^{r} v(x) d x
$$

and

$$
\psi(r)=\int_{0}^{r} \int_{0}^{\min \left(x \tan (\pi / n) \cdot \sqrt{\left.R^{2}-x^{2}\right)}\right.} \frac{h d y d x}{\left(h^{2}+x^{2}+y^{2}\right)^{3 / 2}}=\int_{0}^{r} \varphi(x) d x
$$


where

$$
v(x)= \begin{cases}\frac{h}{3} x \tan \frac{\pi}{n} & \text { for } \quad 0 \leq x \leq R \cos \frac{\pi}{n}, \\ \frac{h}{3} \sqrt{R^{2}-x^{2}} & \text { for } \quad R \cos \frac{\pi}{n} \leq x \leq r,\end{cases}
$$

and

$$
\varphi(x)= \begin{cases}\frac{h x \sin (\pi / n)}{\left(h^{2}+x^{2}\right) \sqrt{x^{2}+h^{2} \cos ^{2}(\pi / n)}} & \text { for } \quad 0 \leq x \leq R \cos \frac{\pi}{n}, \\ \frac{h}{h^{2}+x^{2}} \sqrt{\frac{R^{2}-x^{2}}{R^{2}+h^{2}}} & \text { for } \quad R \cos \frac{\pi}{n} \leq x \leq r .\end{cases}
$$

Those points which are in $\mathscr{C}$ but not $\overline{\mathscr{C}}$ are precisely those with $x$-values in $(\bar{r}, r]$. It will suffice to prove that this region has a higher volume-to-solid-angle ratio than does the whole of $\mathscr{C}$. This will follow if we can show that $v(x) / \varphi(x)$ is increasing. However,

$$
\frac{v(x)}{\varphi(x)}= \begin{cases}\frac{h^{2}+x^{2}}{3} \sqrt{\left(\frac{x}{\cos (\pi / n)}\right)^{2}+h^{2}} & \text { for } 0 \leq x \leq R \cos \frac{\pi}{n} \\ \frac{h^{2}+x^{2}}{3} \sqrt{R^{2}+h^{2}} & \text { for } R \cos \frac{\pi}{n} \leq x \leq r .\end{cases}
$$

It is immediate that this is increasing.

TL9. Let $\psi_{r} \in\left[\hat{\psi}_{2}, \hat{\psi}_{3}\right]$ be as in Proposition $A^{\prime}$. Then

$$
\begin{aligned}
\beta^{\prime}\left(\psi_{r}\right) & =\left(\frac{d \beta}{d \psi}\right)_{\psi=\psi_{r}} \\
& =\frac{1+r^{2}}{\sqrt{6}} .
\end{aligned}
$$

Proof. This result can be calculated directly from Proposition $\mathrm{A}^{\prime}$, but a geometric approach is less tedious. For $\psi_{r} \in\left[\hat{\psi}_{2}, \hat{\psi}_{3}\right], \beta_{r}$ and $\psi_{r}$ are the volume and solid angle, respectively, of a regular, vertex-free, order- 5 SRCC of height 1 , outer radius $1 / \sqrt{2}$, and inner radius $r$. Comparing these with the SRCC corresponding to $r+d r$, we see that the difference consists of the cones over five identical infinitesimal bands like those described in TL6, with $h=1, R=1 / \sqrt{2}$, and $x=r$. The derivative $\beta^{\prime}\left(\psi_{r}\right)$ is simply the volume-to-solid-angle ratio of these cones, which is $\left(1+r^{2}\right) / \sqrt{6}$. 


\section{References}

1. J. H. Conway and N. J. A. Sloane, Sphere Packings, Lattices, and Groups, Springer-Verlag, New York, 1988.

2. Wu-Yi Hsiang, On the sphere packing problem and the proof of Kepler's conjecture, Preprint, 1992.

3. J. H. Lindsey II, Sphere packing in $\mathbf{R}^{3}$, Mathematika 33 (1986), 137-147.

4. J. H. Lindsey II, Sphere packing, Preprint, 1987.

5. D. J. Muder, Putting the best face on a Voronoi polyhedron, Proc. London Math. Soc. (3) 56 (1988), $329-348$.

6. C. A. Rogers, Packing and Covering, Cambridge University Press, Cambridge, 1964.

Received July 18, 1991, and in revised form February 24, 1993, and June 3, 1993. 